\title{
Avanzando la ciencia de enfermería en el Perú: la ciencia del cuidado humano
}

Advancing nursing science in Peru: human caring science

Patrick A. Palmieri ${ }^{1}$, Nataly Membrillo ${ }^{2}$.

\section{RESUMEN}

La ciencia de enfermería enfrenta ahora el reto de avanzar a la siguiente fase de desarrollo, a lo que llamamos 'Ser relevantes a nivel mundial'. Este movimiento implica una variedad de actividades y cambios en la forma que hacemos ciencia, una responsabilidad que debe ser compartida por los científicos en todo el mundo. En el paradigma de la ciencia del cuidado, el método "es siempre subordinado a la esencia del cuidado, y el ethos es el valor basado en la ciencia del cuidado" (Lindberg, von Post, y Eriksson, 2013, p. 100). Esto significa que los estudios de investigación de alta calidad son un resultado importante de la alineación correcta de la ontología, epistemología, metodología y métodos en respuesta a una pregunta de investigación bien definida. La disciplina de la enfermería está "Basada en la ciencia del cuidado; esto a su vez, informa a la profesión. La ciencia del cuidado sirve como punto de partida-moral-filosófico-teórico fundamental para la formación de enfermería, atención al paciente, investigación, e incluso prácticas administrativas" (Watson, 2008, p. 16). En esta tradición, la ciencia de enfermería puede ampliarse para incorporar nuevos métodos de investigación (Phillips, 1990).

Palabras Clave: Cuidado humano, Ciencia de Enfermería

\begin{abstract}
Nursing science now faces the challenge of moving to the next phase of development, which we call 'Be relevant globally'. This movement involves a variety of activities and changes in the way we do science, a responsibility that must be shared by scientists worldwide. In the paradigm of care science, the method "is always subordinate to the essence of care, and the ethos is based on the science of care value" (Lindberg, von Post, and Eriksson, 2013, p. 100). this means that studies of high quality research is an important result of the correct alignment of ontology, epistemology, methodology and methods in response to a question of welldefined research. The discipline of nursing is "Based on the science of care; this in turn, informs the profession. Care science serves as a fundamental to nursing education, patient care, research, philosophicaltheoretical and moral-game and even administrative practices "(Watson, 2008, p. 16). In this tradition, science nursing can be extended to incorporate new research methods (Phillips, 1990).
\end{abstract}

Keywords: Human Care, Nursing Science

${ }^{1}$ DHSc, EdS, MBA, MSN, ACNP, RN, CPHQ, CPHRM, FACHE, FISQua, FAAN, Professor, Walden University, College of Health Sciences, School of Nursing.

${ }^{2}$ Licenciada en Enfermería, Universidad María Auxiliadora.

\section{INTRODUCCION}

Modelos y teorías de enfermería, de Nightingale en adelante, han explicado que la salud no es simplemente la ausencia de enfermedad; se han incorporado muchas dimensiones como la fuerza de vida, patrones y procesos relacionados con la persona, comunidad, y cosmovisiones culturales. Sin embargo, la mentalidad dominante sigue poniendo mucho énfasis e inversión económica sobre la enfermedad en lugar de la salud. Los modernos, contemporáneos y sofisticados sistemas médicos todavía se concentran en la atención de enfermos en vez de brindar un buen cuidado, así, la prevención y la calidad de vida, la muerte, el ser, el devenir, y así sucesivamente (Watson, 2008; p. 228). Hace más de dos décadas, expertos determinaron que la ciencia de la enfermería se encontraba en una coyuntura crítica, con necesidad urgente de desarrollar conocimientos de enfermería relevantes 
para la comunidad global de salud (Meleis, 2010). Considerando la rápida difusión del conocimiento en la profesión enfermería en América del Norte y el contexto global en expansión, Ketefian y Redman, (1997), declararon: "La ciencia de enfermería enfrenta ahora el reto de avanzar a la siguiente fase de desarrollo, a lo que llamamos 'Ser relevantes a nivel mundial'. Este movimiento implica una variedad de actividades y cambios en la forma que hacemos ciencia, una responsabilidad que debe ser compartida por los científicos de los Estados Unidos e internacionalmente "(p. 15). Una vez más, a principios del nuevo milenio los expertos fueron llamados para el desarrollo de un "Modelo holístico universal" para la identidad del profesional de enfermería, como respuesta al impacto de la globalización (Riley, Beal, Levi, y McCausland, 2002). Sin embargo, en este nuevo milenio, hay poca evidencia para demostrar que este desafío ha sidoabordado.

Considerando las diferentes perspectivas sobre la producción de conocimiento (por ejemplo, Boyer, 1990; Fawcett, Watson, Neuman, Walker, y Fitzpatrick, 2001; Glassick, 2000; Schon, 1985, 1995), así como los llamados para reconsiderar la globalización del conocimiento de enfermería basado únicamente en trabajo de campo (Kleinman, Kim, y Basilico, 2013; McKinnon, y Fitzpatrick, 2012), tenemos que establecer las bases teóricas de la enfermería (Morse, 1995) en un contexto global antes de construir la base conceptual (Rodgers, 2000) para la investigación y la práctica. De hecho, las enfermeras peruanas tenemos que llegar a un consenso compatible con colegas extranjeras específicamente sobre la teoría de enfermería y la construcción de una agenda para la acción. Esto significa que tenemos que establecer una agenda guiada, disciplinada $\mathrm{y}$ específica para la investigación de enfermería (Mitchell, 1994). A pesar de la lenta difusión de la teoría de enfermería en América del Sur, tenemos que lograr un paradigma mundial de la enfermería basada en la ontología y definida por la epistemología.

A través de la evaluación del significado de la investigación en enfermería, en el contexto de una filosofía y de la ciencia del cuidado, los investigadores de enfermería peruanos pueden obtener conocimiento de la ciencia del cuidado como una base teórica. Desde el punto de vista de Watson, "La ciencia del cuidado es un campo en evolución-filosófico-ético-epistemológico en el campo de estudio, basado en la disciplina de la enfermería e informado por campos relacionados" (Watson, 2008, p. 18). En el paradigma de la ciencia del cuidado, el método "es siempre subordinado a la esencia del cuidado, y el ethos es el valor basado en la ciencia del cuidado" (Lindberg, von Post, y Eriksson, 2013, p. 100). Esto significa que los estudios de investigación de alta calidad son un resultado importante de la alineación correcta de la ontología, epistemología, metodología y métodos en respuesta a una pregunta de investigación bien definida.

Conocedores como Eriksson (2002), Parse (1990) y Watson (2008) creen que la investigación en enfermería debe evolucionar con métodos clínicos derivados de la teoría de enfermería. Con el fin de definir la enfermería como disciplina autónoma, se requiere de la investigación básica. Según Asp y Fagerberg (2005) "La ambición de la investigación básica en la ciencia del cuidado es la creación de fundamentos teóricos que describen y explican el significado del cuidado y las condiciones para el cuidado" (p. 2). Esto significa que las enfermeras debemos establecer nuestro fundamento filosófico con hipótesis coherentes en lo ontológico, epistemológico y metodológicos. Entonces, ¿Qué significa esto en el contexto del avance de la ciencia de la enfermería peruana? Según Mitchell (1994), ya sea considerando a la hermenéutica o fenomenología, los investigadores tienen que entender primero su perspectiva teórica antes de seleccionar un método apropiado que está alineado con un método específico. Por lo tanto, antes de considerar la metodología cualitativa asociada a la enfermería basada en evidencia o uno de los cuatro principales tipos de diseños de investigación cualitativa (la fenomenología, la etnografía, la teoría fundamentada, y los estudios de casos), la pregunta de investigación debe ser definida en base a la teoría de enfermería.

Si regresamos a Watson a contemplar esta pregunta, nos encontramos con "La ciencia del cuidado es el punto de partida para la enfermería como un campo de estudio que ofrece una base disciplinaria distinta de la profesión; proporciona, moral, valor - meta paradigma- una narrativa para la ciencia y sus fenómenos humanos, con enfoque de cuidado - sanación - persona - naturaleza universo. Se vuelve a introducir el espíritu y las dimensiones sagradas en nuestro trabajo, vida y en el mundo" (Watson, 2008, p. 16). De hecho, aquellas preguntas de investigación derivadas de la ciencia del cuidado se basan en la teoría de enfermería. Expertos señalan que el énfasis de enfermería experimenta conocimiento derivados de la práctica y el conocimiento científico derivado de la investigación. Aunque Eriksson (2002) señala que el fenómeno de cuidar, diferencia la profesión de enfermería de otras profesiones como las ciencias sociales y las ciencias médicas, sin 
embargo, estos pueden ser complementarios. Por ejemplo, considere que la ciencia cuidado es complementaria a la medicina que es la ciencia del curar. En este contexto, las ciencias de enfermería y la medicina como el cuidado y la curación de son sinónimo de los productos, balanceado por la armonía del ying y el yang.

Para concluir, la disciplina de la enfermería está "Basada en la ciencia del cuidado; esto a su vez, informa a la profesión. La ciencia del cuidado sirve como punto de partida-moral-filosófico-teórico fundamental para la formación de enfermería, atención al paciente, investigación, e incluso prácticas administrativas" (Watson, 2008, p. 16). En esta tradición, la ciencia de enfermería puede ampliarse para incorporar nuevos métodos de investigación (Phillips, 1990). El paradigma de la ciencia del cuidado permite a los enfermeros considerar cuestiones pertinentes en el contexto de la práctica de la Enfermería Peruana, tales como: ¿Es válido el juicio clínico como prueba? ¿La teoría de enfermería se considera como evidencia? ¿Percepciones personales, observaciones y experiencias cuentan cómo evidencia? ¿Cómo está evidencia se produce desde y dentro del entorno en la atención del paciente?. Siguiendo la lógica de Connor (1996), las enfermeras peruanas tienen que entablar un diálogo concreto para avanzar en la investigación y la práctica de enfermería en el contexto de la ciencia y la filosofía del cuidado humano. Ya que, sin incorporar la ciencia del cuidado humano y sus diez factores de caritas, las enfermeras pueden no haber estado practicando la profesión de enfermería más bien solo sirviendo como técnicas o trabajadoras con habilidades en el imperioso marco de la ciencia médica tecnocurativa (Watson,2008, p. 3). Las enfermeras contribuyen a fortalecer el sistema de salud de muchas maneras, incluyendo: las principales iniciativas de políticas de salud; contribuyen al desarrollo de los servicios; la supervisión y el desarrollo de otros miembros del equipo; trabajan con y defienden a los pacientes, sus cuidados y comunidades; y la recolección de datos e informar el desarrollo de pruebas científicas y políticas. Las decisiones que la enfermera realiza en el día a día marcan una gran diferencia significativa en la eficiencia y la efectividad de todo el sistema (Internacional Nursing Review, ICN, 2016, p. 12)

\section{CORRESPONDENCIA:}

Nataly Membrillo Pillpe.

Universidad Maria Auxiliadora

Avenida Canto Bello 431, San Juan de Lurigancho. Teléfono: 998469500

E-mail: enfermería@uma.edu.pe

\section{REFERENCIAS BIBLIOGRAFICAS}

1. Asp, M., \& Faberberg, I. (2005). Developing concepts in caring science based on a lifeworld perspective. International Journal of Qualitative Methods, 4(2), 1-10.

2. Boyer, E. L. (1990). Scholarship reconsidered: Priorities of the professoriate. Menlo Park, CA: Carnegie Foundation.

3. Connors, M. J. (1996). Expanding the dialogue on praxis in nursing research and practice. Nursing Science Quarterly, 11(2), 51-55.

4. Eriksson, K. (2002). Caring science in a new key. Nursing Science Quarterly, 15(1), 61-65.

5. Farmer, P., Kleinman, A., Kim, J. Y., \& Basilico, M. (2013). Reimagining global health: An introduction. Berkeley, CA: University of California Press.

6. Fawcett, J., Watson, J., Neuman, B., Walker, P. H., \& Fitzpatrick, J. (2001). On nursing theories and evidence. Journal of Nursing Scholarship, 33(2), 115-119.

7. Glassick, C. E. (2000). Boyer's expanded definitions of scholarship, the standards for assessing scholarship, and the elusiveness of the scholarship of teaching. Academic Medicine, 75,877-880.

8. Ketefian, S., \& Redman, R. W. (1997). Nursing science in the global community. Journal of Nursing Scholarship, 29(1), 11-15.

9. Lindberg, S., von Post, I., \& Eriksson, K. (2013). Hermeneutics and human interplay: A clinical caring science research method. International Journal of Qualitative Methods, 12 , 99-112. http://ejournals.library.ualberta.ca/index.php/I JQM/article/view/17669/14876

10. Mitchell, G. J. (1994). Discipline-specific inquiry: The hermeneutics of theory-guided nursing research. Nursing Outlook, 42(5), 224-228.

11. Meleis, A. I. (2010). Transitions theory: Middle range and situation specific theories in nursing research and practice. New York, NY: Springer Publishing.

12. McKinnon, T., \& Fitzpatrick, J. (2012). Global service-learning in nursing. Washington, DC: National League for Nursing.

13. Morse, J. (1995). Exploring the theoretical basis of nursing using advanced techniques for concept analysis. Advances in Nursing Science, 17(3), 31-46.

14. Parse, R. R. (1990). Parse's research methodology with an illustration of the lived experience of hope. Nursing Science Quarterly, 3, 1-17. 
15. Phillips, J. R. (1990). New methods of research, beyond the shadows of nursing science. Nursing Science Quarterly, 3, 1-2.

16. Riley, J. M., Beal, J., Levi, P., \& McCausland, M. P. (2002). Revisioning nursing scholarship. Journal of Nursing Scholarship, 34(4), 383389.

17. Rodgers, B. L. (2000). Philosophical foundation of concept development. In B. L. Rodgers \& K. A. Knafl (Eds.), Concept development in nursing: Foundation, techniques and application (pp. 7-37). London: Saunders.

18. Schon, D. A. (1983). The reflective practitioner: How professionals think in action. New York, NY: Basic Books.
19. Schon, D. A. (1995). Knowing in action. The new scholarship requires a new epistemology. Change, 27(6), 27-34.

20. Watson, J. (2008). Nursing: The philosophy and science of caring. Boulder, CO: University Press of Colorado.

21. International Perspectives. International Nursing Review. (2016). 63: 7-14. International Council of Nurses doi: 10.1111/inr.12253.

Recibido: 23/03/2016

Aceptado: 22/06/2016 